\title{
Theme issue shoulder instability
}

\author{
Klaus Bak ${ }^{1} \cdot$ Pietro Randelli ${ }^{2}$ Olaf Lorbach ${ }^{3}$
}

Received: 15 January 2016 / Accepted: 18 January 2016 / Published online: 25 January 2016

(C) European Society of Sports Traumatology, Knee Surgery, Arthroscopy (ESSKA) 2016

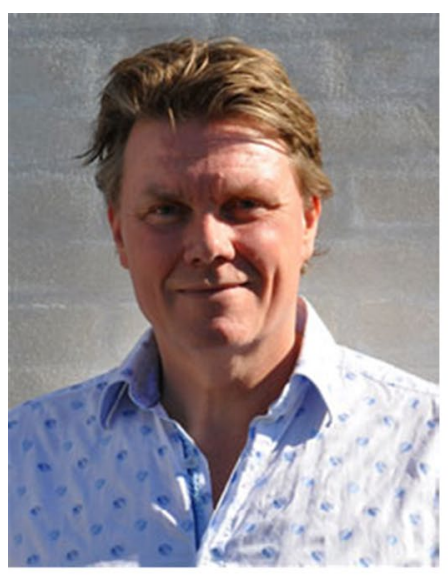

Klaus Bak

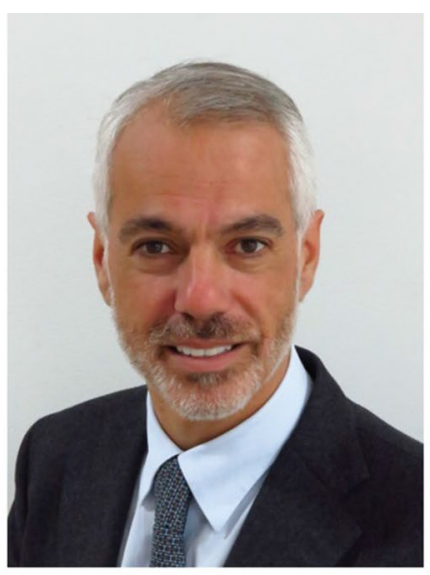

Pietro Randelli

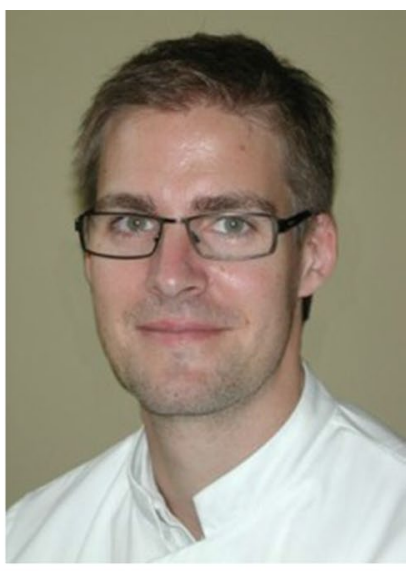

Olaf Lorbach
When we were young residents, we were taught that a Hill-Sachs lesion of the humeral head was a normal finding in glenohumeral instability and that it had no clinical significance. This was before MR technology, arthroscopic

Klaus Bak

skulderbak@gmail.com

Pietro Randelli

pietro.randelli@unimi.it

Olaf Lorbach

olaf.lorbach@gmx.de

$1 \quad$ Arthroscopic and Reconstructive Shoulder Sugery, Aleris Hamlet Parken, Øster Allé 42, 2nd flooor, 2100 Copenhagen $\varnothing$, Denmark

2 Dipartimento di Scienze Biomediche per la Salute, Università degli Studi di Milano, Direttore U.O. Ortopedia 2, IRCCS Policlinico San Donato, Milan, Italy

3 Department of Orthopaedic Surgery, Saarland University, Kirrberger Str., Geb. 37/38, 66421 Homburg, Saar, Germany reconstructive surgery, 3D CT reconstructions and the possibility to make animations that can mimic the effect of the location of the Hill-Sachs lesion. It was also the time where the indication for stabilizing surgery was at least six dislocations and that open procedures had a low failure rate but also in some cases with a significant reduction in external rotation. Other open procedures like Bristow-Latarjet were known to have low recurrence rate but showed the same risk of reducing the ability to fully externally rotate the arm overhead, which is a disadvantage for the active overhead athlete. In the 1990s arthroscopic repair became more and more popular possibly due to the fact that the pathology could be assessed more precisely and because the repair aimed at being more anatomic, but at the very end of the last century, the drawback was initially associated with a recurrence rate of over $30 \%$. Today, we know that the number of recurrences not only increases the risk of osteoarthritis but also makes simple arthroscopic Bankart repair less effective. During the past 15 years, we have been moving from a discussion between open Bankart and arthroscopic 
Bankart towards a more differentiated menu of treatment options where a more precise correction of structural pathology seems to improve the success rate of surgery. In the light of the expected failure rate of soft tissue reconstructive procedures, the Latarjet-Patte-Bristow procedure and other bone transfer procedures become more and more common and show lower failure rates. Correcting bony instability is at present the most common issue discussed, which is reflected by the proportion of papers in this issue that highlights pro and cons of these procedures. The recurrence rate seems to be lower, but some of the papers in this issue, however, points to the fact that some of these procedures are technically demanding and have a steep learning curve, and that serious complications (nerve injury etc.), which you do not see with soft tissue procedures, may be a challenge even for the advanced shoulder surgeon.

Whereas it has been clearly shown in a number of randomized studies that Bankart repair is superior to non-operative treatment in young active first-time dislocators, in daily life many patients prefer non-operative treatment as the initial treatment. The challenging part of our clinical work is to guide the patient towards the treatment with the best possible outcome, less invasiveness and good long-term prognosis. Whereas traumatic instability is well understood and a relation between structural pathology and intervention seems to be present, the picture is more blurred when it comes to microtraumatic instability in overhead athletes. The pathology is different, and with coexisting pathologies and underlying dysfunctions, thus simply repairing the pathology is rarely the primary solution to make the athlete return to pre-injury activity and performance. The complex pattern of the disabled overhead athlete's shoulder involving glenohumeral instability may be treated with more success correcting the dysfunctions that eventually lead to instability. The scapula plays a central role in this condition but should also be kept in mind in other patients with glenohumeral instability.

In the current theme issue, we aimed at covering all aspects of today's knowledge on glenohumeral instability. With the help of authors submitting high-relevance papers to update on this matter as well as with invited review papers regarding major subjects, we believe that we have succeeded in showing as many colours of the palette that was possible. 